\title{
Low-Dimension Nanomaterial-Based Sensing Matrices for Antibiotics Detection: A Mini Review
}

\author{
Yucan Dong ${ }^{1,2}$, Fengting $\mathrm{Li}^{1,2}$ and Ying Wang ${ }^{1,2 *}$ \\ ${ }^{1}$ State Key Laboratory of Pollution Control and Resources Reuse, College of Environmental Science and Engineering, Tongji \\ University, Shanghai, China, ${ }^{2}$ Shanghai Institute of Pollution Control and Ecological Security, Shanghai, China
}

\section{OPEN ACCESS}

Edited by:

Mingshui Yao,

Kyoto University, Japan

Reviewed by:

Liwei Wang

Guangxi University, China

Xiaochuan Duan,

Xiamen University, China

*Correspondence:

Ying Wang

yingwang@tongji.edu.cn

Specialty section:

This article was submitted to

Nanoscience,

a section of the journal

Frontiers in Chemistry

Received: 10 February 2020 Accepted: 28 May 2020

Published: 24 July 2020

Citation:

Dong Y, Li F and Wang Y (2020) Low-Dimension Nanomaterial-Based

Sensing Matrices for Antibiotics

Detection: A Mini Review.

Front. Chem. 8:551.

doi: 10.3389/fchem.2020.00551
Antibiotics, a kind of secondary metabolite with antipathogen effects as well as other properties, are produced by microorganisms (including bacterium, fungi, and actinomyces) or higher animals and plants during their lives. Furthermore, as a chemical, an antibiotic can disturb the developmental functions of other living cells. Moreover, it is impossible to avoid its pervasion into all kinds of environmental media via all kinds of methods, and it thus correspondingly becomes a trigger for environmental risks. As described above, antibiotics are presently deemed as a new type of pollution, with their content in media (for example, water, or food) as the focus. Due to their special qualities, nanomaterials, the most promising sensing material, can be adopted to produce sensors with extraordinary detection performance and good stability that can be applied to detection in complicated materials. For low-dimensional (LD) nanomaterials, the quantum size effect, and dielectric confinement effect are particularly strong. Therefore, they are most commonly applied in the detection of antibiotics. This article focuses on the influence of LD nanomaterials on antibiotics detection, summarizes the application of LD nanomaterials in antibiotics detection and the theorem of sensors in all kinds of antibiotics detection, illustrates the approaches to optimizing the sensitivity of sensors, such as mixture and modification, and also discusses the trend of the application of LD nanomaterials in antibiotics detection.

Keywords: nanomaterials, antibiotics detection, low-dimension, fluorescent, electrochemical

\section{INTRODUCTION}

Since their discovery in 1929, antibiotics have been extensively adopted for many decades. At present, there is abundant research on the content of antibiotics in water bodies and food as well as the transportation, transformation, and degeneration patterns of antibiotics in nature. Although most antibiotics do not have long half-lives, they can be regarded as chronic organic pollutants due to their long-term and lasting use. Antibiotics are harmful to the human body as they can alter the microbial community inside it, disturb the human metabolism, and produce antibiotic-resistant genes and antibiotic-resistant bacteria in the environment (Kuemmerer, 2009; Ben et al., 2019). The concentration of antibiotics in wastewater ranges from a few ng to tens of thousands of $\mu g$. Nonetheless, antibiotics are rarely detected in other environmental media such as air, and the residue in soil mainly comes from irrigation (Mohammad-Razdari et al., 2019a).

At present, detection of the amount of antibiotics has already achieved a relatively low limit of quantitation. For example, by combining solid-phase extraction with liquid chromatography, 
the quantitation limit can be taken to an order of magnitude lower than $10^{-12} \mathrm{M}$. However, the instrument mentioned above is bulky and difficult to operate (Netea et al., 2019). In terms of innovation, nanomaterials have four distinctive properties, namely their surface effect, quantum size effect, quantum tunnel effect, and dielectric confinement effect. LD materials have a high specific surface area, with quick electron conduction. Moreover, the high abundance of surface defects helps them to emit highintensity fluorescence, which is an excellent characteristic for both the detector of the sensor and the signal sensing module (Coleman et al., 2006). Sensors produced with this kind of material are easy to operate, quick in detection, and highly sensitive, making them the primary direction of development in this field at present. This article summarizes work on the application of 0 -, 1-, and 2-dimensional nanomaterials in antibiotics detection, respectively, explores the application mechanism of nanomaterials, and puts forward the development trend in this field.

\section{THE ROLE OF NANOMATERIALS IN ANTIBIOTICS DETECTION}

The concept of nanomaterials began with nanocrystaline research by Gleiter and Marquardt (1984), Gleiter (1989), Gleiter (2000), and since then, nanomaterials have gradually become a hot topic of research. Nanomaterials can be divided into 0-dimensional (0D), 1-dimensional (1D), 2-dimensional (2D), and 3-dimensional (3D) in terms of dimensional characteristics. Dimensions refer to the number of dimensions where the material is not within the size boundary of $0.1-100 \mathrm{~nm}$. Because their dimensions and structures are different, the sensitivity of sensors made of different nanomaterials is different according to the surface effect and the properties of the dielectric region (Figure 1).

The types of nanomaterials adopted for the determination of antibiotics are as follows. Among them, 0D materials include nanoclusters (Zang et al., 2013), nanoparticles (Wu et al., 2018), and quantum dots (QDs) (Malik et al., 2019); the 1D materials include nanowires (Shad et al., 2019), nanotubes (Xiao et al., 2007), and nanorods (Roushani and Ghanbari, 2018); the 2D materials include nanoflakes (Zeng et al., 2018) and nanofilms (Velusamy et al., 2018).

There are two common types of antibiotic detection sensors at present. One is an electrochemical (EC) sensor (Liu et al., 2019), and the other is a fluorescence (FL) sensor (Peng et al., 2018). The common characteristics of these two types of sensors are fast response, convenient operation, portability, and a low limit of detection (LOD). An EC sensor reflects the concentration of the measured object with electrical signals, so nanomaterials are commonly used to promote conductivity. Nanomaterials are often adopted in an FL as fluorescent materials or as fluorescence quenching materials, such as carbon quantum dots (CQDs) (Chen et al., 2018). The details of the types, detection limits, and measurement properties of nanomaterial sensors are given in Table 1.

In the next section, we will introduce the application of $0 \mathrm{D}$, $1 \mathrm{D}$, and $2 \mathrm{D}$ nanomaterials in the two types of sensors, FL and EC. We will then summarize and forecast the status and trends of research in related fields in the third section.

\section{LD NANOMATERIAL-BASED SENSORS FOR ANTIBIOTICS DETECTION}

\section{LD Nanomaterial-Based FL Sensors}

FL sensors have high selectivity because the occurrence and quenching of fluorescence have specificity. Therefore, the use of FL sensors for the detection of antibiotics in complex media (such as milk, honey, etc.) has high research value, and it is one of the hot spots in the research field in recent years.

The most common 0D nanomaterials adopted in FL sensors are QDs (Liu et al., 2009). This is because of the long-term photostability, high-quantum yield, narrow emission, and broad excitation spectra of CdX QDs (Leptihn et al., 2010; Hou et al., 2011). QDs are generally composed of elements from groups III to $\mathrm{V}$ or II to IV in the periodic table of the elements (Zhang and Wei, 2016). QDs were applied as early as 2006. At that time, CdX $(X=S$, Se, etc.) QDs were mainly adopted (Qie Gen et al., 2007; Wang et al., 2009, 2010).

For example, Ding et al. adopted CdSe QDs and competitive fluorescence-linked immunosorbent assay (cFLISA) for the detection of sulfamethazine (Ding et al., 2006). QDs were applied as a fluorescence label in the cFLISA method. The combination of the cFLISA method and QDs has great specificity, so it can be used for the determination of antibiotic residues in chicken muscle. The LOD is as low as $3.6 \times 10^{-9} \mathrm{M}$. In addition to QDs, other nanoparticles are also widely adopted in the design of antibiotic sensors. Berlina et al. integrated lateral flow assay with fluorescent labels, which provides the opportunity to achieve simple and sensitive control of milk contamination by chloramphenicol. This method also illustrates the high specificity of the combination of QDs and immunization methods. The LOD of chloramphenicol is $9.3 \times 10^{-9} \mathrm{M}$ (Berlina et al., 2013). NPs are also widely adopted in the design of antibiotic sensors. Mesoporous silica nanoparticles are an example of non-metal oxide nanoparticles that can be used (Wang C. et al., 2018). Wang et al. adopted aggregation-induced emission luminogens to functionalize silica nanoparticles. Because of the restricted intramolecular vibration and rotation in rigid silica networks, the material emitted strong blue fluorescence, which showed highly sensitive fluorescence-quenching responses toward nitrofuran antibiotics. The LOD of nitrofurazone reached $7.2 \times 10^{-6} \mathrm{M}$.

1D nanomaterials have relatively few applications in the fluorescence detection of antibiotics. 1D nanomaterials like nanotubes or nanorods are more applied in EC sensors, which will be described in detail in the next section. Liu et al. developed a semiconductor of $\mathrm{ZnO}$ nanomaterials as a fluorescence sensor without leakage toxicity. $\mathrm{ZnO}$ nanomaterials have desirable optical and electronic properties, a large excitation binding energy of $60 \mathrm{~m} \mathrm{eV}$, and a wide bandgap. Liu adopted molecular 


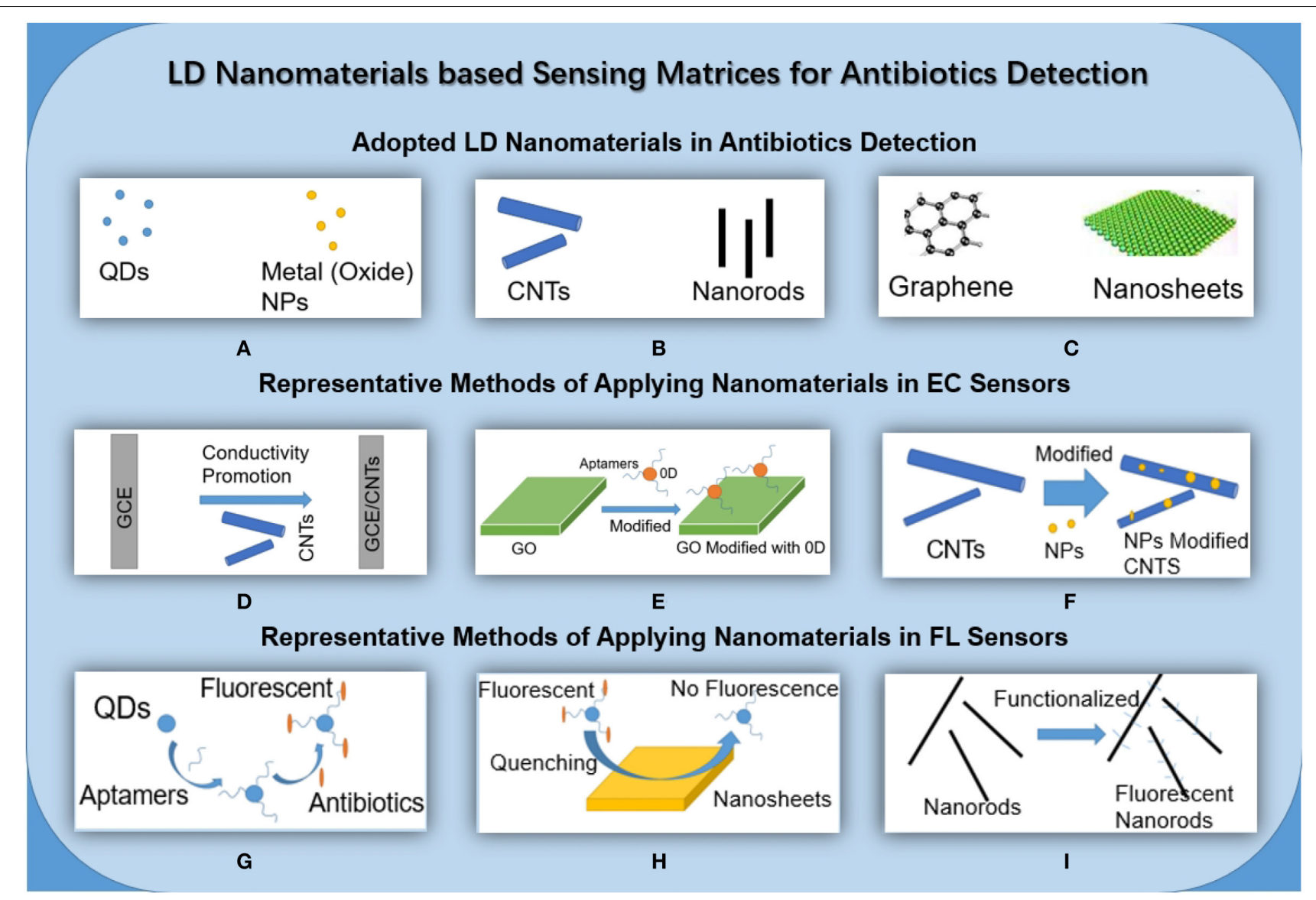

FIGURE 1 | Schematic diagram of LD nanomaterials for EC and FL. (A). OD nanomaterials commonly used in the construction of sensors for antibiotic detection. (B) 1D nanomaterials commonly used in the construction of sensors for antibiotic detection. (C) 2D nanomaterials commonly used in the construction of sensors for antibiotic detection. (D) A common strategy for applying nanomaterials in EC sensors: modify the electrode with CNTs to improve electron conductivity. (E) A common strategy for applying nanomaterials in EC sensors: use GO in conjunction with OD nanomaterials, which improves electrical conductivity, and modify the electrode. (F) A common strategy for applying nanomaterials in EC sensors: use NPs to modify carbon nanotubes and further improve their biocompatibility and conductivity. (G) A common strategy for applying nanomaterials in FL sensors: QDs combined with aptamers and functionalized that recognize specific antibiotics and fluoresce. (H) A common strategy for applying nanomaterials in FL sensors: as a detection platform, the 2D nanosheet has a fluorescence quenching effect. (I) A common strategy for applying nanomaterials in FL sensors: functionalized nanorods fluoresce in response to specific antibiotics.

imprinting polymer technology and $\mathrm{ZnO}$ nanomaterials to realize specific selectivity, high stability, and easy operation, which exhibited promising application in sensors (Liu et al., 2020).

Due to the superiority of $2 \mathrm{D}$ nanomaterials on the macro scale, they are easily adopted in sensing platforms. For example, Qin et al. made a fluorescent sensing platform for bleomycins by using $\mathrm{WS}_{2}$ nanosheets. In the presence of $\mathrm{Fe}(\mathrm{II})$, bleomycin forms a BLM.Fe(II) complex that reacts with oxygen to generate a BLM·Fe(III)OOH species. BLM.Fe(III)OOH can catalyze the incision of DNA. $\mathrm{WS}_{2}$ nanosheets exhibit different affinity toward single-stranded DNA (ssDNA) with different length and excellent fluorescence quenching ability. BLM catalyzes the incision of long ssDNA and restores fluorescence, Different fluorescence intensities correspond to different BLM concentrations. Using the above-described detection principle, the LOD of BLM is $3.0 \times 10^{-10} \mathrm{M}$. Using rolling circle amplification to amplify the signal is an important factor in this method, and using ssDNA as a signal has good biological activity (Qin et al., 2015).

According to the literature summarized above, the nanomaterials used in FL are mainly $0 \mathrm{D}$ and 2D. $0 \mathrm{D}$ materials like QDs are rich in surface defects and are easy to modify and synthesize. They will emit high-intensity fluorescence when they encounter specific substances, which has good application value for antibiotics detection in complex environments. Compared with CdX QDs, CQDs are less biotoxic and have better biocompatibility. $2 \mathrm{D}$ materials are more suitable for building platforms, as they have good fluorescence quenching performance.

\section{LD Nanomaterial-Based EC Sensors}

The characteristics of EC sensors are that they are fast, low-cost, high-sensitivity, and relatively portable, and they have hence been one of the development directions in environmental pollutant detection in recent years. In this part, we will mainly introduce 
TABLE 1 | Characteristics of the different nanomaterials applied.

\begin{tabular}{|c|c|c|c|c|}
\hline Type & Nanomaterials & Role of nanomaterial & Analyte & References \\
\hline FL & OD CdSe-ZnS QDs & Sensitivity enhancement & Sulfamethazine & Ding et al., 2006 \\
\hline EC & 1D MWCNTs & Sensitivity, selectivity enhancement & Oxytetracycline & Vega et al., 2007 \\
\hline EC & OD/1D AuNPs/SWCNTs & Sensitivity and stability enhancement & Chloramphenicol & Xiao et al., 2007 \\
\hline $\mathrm{FL}$ & OD CdSe-ZnS QDs & Sensitivity enhancement & Enrofloxacin & Chen et al., 2009 \\
\hline $\mathrm{FL}$ & OD CdTe QDs & Sensitivity enhancement & Etimicin & Wang et al., 2010 \\
\hline EC & 2D GOs & Sensitivity and stability enhancement & Alpha fetoprotein & Wei et al., 2010 \\
\hline EC & OD/1D AuNCs/AuNRs & Selectivity enhancement & Ofloxacin & Zang et al., 2013 \\
\hline EC & 1D/2D MWCNTs/GOs & Simplicity, stability enhancement & Azithromycin & Zhang et al., 2013 \\
\hline EC & 2D CuO nanosheets & Sensitivity enhancement & Vancomycin & Khataee et al., 2014 \\
\hline EC & 2D rGO nanosheet & Sensitivity enhancement & Rifampicin & Rastgar and Shahrokhian, 2014 \\
\hline EC & OD gold@silver nanoparticles & Sensitivity enhancement & Kanamycin & Zengin et al., 2014 \\
\hline $\mathrm{FL}$ & 2D $\mathrm{WS}_{2}$ nanosheet & Selectivity and sensitivity enhancement & Bleomycin & Qin et al., 2015 \\
\hline EC & 1D/2D MWCNTs/MoS 2 nanosheets & Reproducibility and stability enhancement & Chloramphenicol & Govindasamy et al., 2017 \\
\hline EC & OD AuNPs & Sensitivities and specificity & Chloramphenicol & Huang et al., 2018 \\
\hline $\mathrm{FL}$ & OD CdTe QDs & Sensitivity enhancement & Sulfadiazine & Chen et al., 2018 \\
\hline EC & OD/2D g- $\mathrm{C}_{3} \mathrm{~N}_{4}$ QDs/rGOs & Sensitivity enhancement & Sulfadimethoxine & Dang et al., 2018 \\
\hline EC & 2D graphene & Stability and repeatability enhancement & Erythromycin & Huang et al., 2018 \\
\hline EC & OD Silver NPs & Speed and sensitivity enhancement & Ampicillin & Rosati et al., 2019 \\
\hline EC & OD CdS QDs & Selectivity and sensitivity enhancement & Chloramphenicol & Wang Y. et al., 2018 \\
\hline $\mathrm{FL}$ & $\mathrm{OD} \mathrm{g}-\mathrm{C}_{3} \mathrm{~N}_{4} \mathrm{QDs}$ & Sensitivity enhancement & Amikacin & Hassanzadeh et al., 2019 \\
\hline EC & OD/1D AuNPs/MWCNTs & Sensitivity enhancement & Oxytetracycline & He et al., 2019 \\
\hline EC & OD/2D AuNPs/GOs & Speed and sensitivity enhancement & Penicillin & Mohammad-Razdari et al., 2019b \\
\hline EC & OD/2D AuNPs/rGOs & Reproducibility and selectivity enhancement & Sulfadimethoxine & Mohammad-Razdari et al., 2019a \\
\hline
\end{tabular}

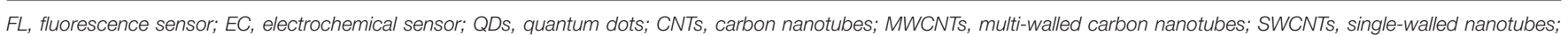
GOs, graphene oxides; AuNCs, gold nanoclusters; AuNPs, gold nanoparticles; AuNRs, gold nanorods; rGOs, redox graphene oxides.

the ways in which $0 \mathrm{D}, 1 \mathrm{D}$, and $2 \mathrm{D}$ nanomaterials are used to improve the performance of EC sensors.

For 0D materials, QDs can be taken as an example. QDs can be used as stabilizers (Xu et al., 2018). Besides being adopted as stabilizers, Dang et al. adopted $\mathrm{g}-\mathrm{C}_{3} \mathrm{~N}_{4}$ QDs modified with redox graphene oxides (rGOs) in photoelectric sensors to measure sulfadimethoxine (SDM). g- $\mathrm{C}_{3} \mathrm{~N}_{4}$ QDs possess good optical and electrical properties as compared to other carbon-based QDs (Dang et al., 2018). Due to their lower intrinsic conductivity, they need to be modified with materials with high conductivity and hydrophilic ability for better performance in the detection of antibiotics. By modifying g- $\mathrm{C}_{3} \mathrm{~N}_{4}$ QDs with rGOs, a linear calibration range for SDM of $5.0 \times 10^{-10} \mathrm{M}-1.4 \times 10^{-15} \mathrm{M}$ was obtained, with a LOD of $1.0 \times 10^{-10} \mathrm{M}$.

1D materials perform well in EC sensors. The general method of applying 1D nanomaterials in antibiotics sensing is through electrode surface modifications (Vega et al., 2007). Zhang et al. combined the hydrophilic properties of rGOs and the excellent electronic and antifouling properties of multi-walled carbon nanotubes (MWCNTs) for azithromycin detection. Compared with a bare glassy carbon electrode (GCE), the GCE modified with MWCNTs has a larger active surface area. The LOD of tetracyclines in a water sample was $1.1 \times 10^{-9}-6 \times 10^{-9} \mathrm{M}$ under pre-concentration (Zhang et al., 2013). P-type semiconductor-based transition metal oxide nanoparticles have received much attention due to their excellent physicochemical properties, which make them suitable for catalysis, energy storage, and electrochemical conversion applications. The integration of metal oxides and their synergistic effects enhance selectivity and activity. Chen et al. synthesized a CuO NPs@MWCNTs nanocomposite (Chen et al., 2019). AuNPs have also been introduced to anchor on MWCNTs, not only providing a large number of biological binding sites but also effectively improving the electron transfer rate (He et al., 2019).

In terms of cost or difficulty of preparation, carbon nanotubes are excellent choices, but nanorods and nanowires also have unique properties due to their morphologies. Nanowires can be easily synthesized to achieve a multisegment structure, which will enable the nanowire to be used for multifunctional applications, especially as high sensitivity, multitasking electrochemical biosensors ( $\mathrm{Li}$ et al., 2019). Nanorods have a relatively small specific surface area but also have excellent electronic conductivity and are more stable.

$0 \mathrm{D}, 1 \mathrm{D}$, and $2 \mathrm{D}$ nanomaterials are used to improve the performance of electrochemical sensors. Herein, by viewing the reported studies, it can be seen that combinations of 0D (such as NPs) and 2D (rGOs) nanoparticles have attracted a lot of attention in this research field. Mohammad et al. fabricated an electrochemical aptamer-based biosensor on a pencil graphite electrode (PGE). The bare PGE was modified with rGOs and AuNPs for SDM determination. This optimizes the linear 
range of the electrode as well as stability and reproducibility (Mohammad-Razdari et al., 2019b).

\section{CONCLUSION AND OUTLOOK}

\section{Supplementary Notes on Methods of Applying LD Nanomaterials in Antibiotic Detection}

For 0D materials, QDs are often used as fluorophores with excellent performance, and metal or metal oxide nanoparticles are often used to modify other materials to further improve electronic conductivity. For 1D materials, nanotubes, nanowires, and nanorods are often used to enhance sensor performance, or to form microelectrode arrays; 2D materials, meanwhile, are more convenient for constructing platforms (Wang et al., 2017; He and Yan, 2018). Sensitivity is usually enhanced by means of signal amplification and enhancement (Wei et al., 2010). In order to improve the performance of the sensor, combination of nanomaterials in various dimensions is a very common method, such as using AuNPs to modify rGOs to improve the electronic conductivity of the sensor, which promotes the stability and sensitivity of the sensor. Good electron transfer capabilities and conductivity can also promote selectivity (Munawar et al., 2018).

When designing sensors, fluorescence immunoassay (FIA) is one of the promising technologies for antibiotics detection based on LD nanomaterials. In order to cope with complex environments, the sensors need to have good selectivity (Govindasamy et al., 2017). For example, FIA, a type of immunoassay, has two models: competitive type and sandwich type. The competitive type refers to a competitive mechanism that enables unlabeled antigens to compete with labeled antigens by binding to a limited antibody. For the competitive type, unlabeled antigens, and labeled antigens compete with antibodies that are bound to QDs. The fluorescence intensity varies with different antigen concentrations (Chen et al., 2009; Zhu et al., 2011).

For the sandwich type, the reaction principle is illustrated as follows. An excess of antibody is fixed on the immune reaction carrier, and subsequently, a certain amount of antigen is added. After the immune reaction, an excess of labeled antibody is added to form a "sandwich" immune complex. The more antigens in the sample and the more labeled antibodies bound, the stronger the labeled fluorescent signal of the sandwich immune complex is. Zengin et al. adopted this method and made a sensor on the basis of gold and silver nanoparticles. Accordingly, the LOD of kanamycin can reach $3.4 \times 10^{-12} \mathrm{M}$ (Zengin et al., 2014).

This method can be adopted in high-precision detection of antibiotics in complex media; such applications have been introduced above in the $0 \mathrm{D}$ section.

\section{Summary and Outlook}

Due to their different morphologies and dimensions, different nanomaterials have different beneficial properties.

The development of antibiotics sensors in the next few years can be roughly divided into the following directions: the pursuit of high-sensitivity detection, the pursuit of low-cost and fast onsite detection, and the pursuit of multi-functional integration. Among them, the gradual realization of low-cost and rapid monitoring should be the most noteworthy, and this fits with the advantages of EC. According to the 57 articles cited in this article, we concluded that, in FL, OD is currently used most, followed by $2 \mathrm{D}$, and $1 \mathrm{D}$ is used least. $0 \mathrm{D}$ is represented by various types of QDs that can be used as fluorophores. In EC, 1D is the most used type, and $2 \mathrm{D}$ and $0 \mathrm{D}$ are used relatively little. In the future, researchers who want to improve FL performance can pay more attention to new quantum dots and NPs, and researchers who want to improve EC performance can pay more attention to materials with good electron conduction properties such as CNTs. At the same time, the performance improvement brought about by NP modification of other nanomaterials should also be considered. Finally, when the wish is to build a platform, 2D materials have advantages in terms of morphology. At present, there are few related studies, and in the coming decades, these may be hot research topics (Rastgar and Shahrokhian, 2014; Kokulnathan and Chen, 2020; Liu et al., 2020; Roushani et al., 2020).

In order to further optimize the performance of antibiotics sensors based on LD nanomaterials, the following suggestions are proposed according to the application status:

1. Fluorescence immunoassay method can be better applied to the detection of low levels of antibiotics in complex media, such as chicken muscle, milk, and honey. This is because quantum dots can be well combined with antibody immunoassay. This method has great specificity, lower LOD, and good biocompatibility. In order to achieve specific functions for various antibiotics, different immunoassays combined with QDs need to be designed.

2. Pay attention to the portability, fastness of measurement, and cooperation with smart devices (Tian et al., 2015). Taking the research by $\mathrm{Yu}$ et al. As an example, the electrodes were printed by screen printing technology to achieve rapid response measurement within $30 \mathrm{~s}$ (Yu et al., 2019). The research of Wang et al. in 2020 realized online monitoring through the color selection application of the mobile phone and the fluorescence reaction. These studies have good application value.

3. Build a platform that can detect multiple antibiotics at the same time. The platformization of the sensor depends on 2D nanomaterials or MOF. MOF is often used in combination with metal nanoparticles to obtain better performance. To achieve the simultaneous measurement of multiple antibiotics, microarray electrode technology can achieve this complex function.

4. In addition to the combination with MOF, the combination of NPs and other materials can also achieve better performance, which is a breakthrough direction for high-precision research.

\section{AUTHOR CONTRIBUTIONS}

YW and FL supervised this work. YW established the conceptualization of this work and acquired the funding. YD did 
research on methodology, collected the data, edited, and wrote this review. All of the authors read and approved the manuscript.

\section{FUNDING}

This work was supported by the National Natural Science Foundation of China (NSFC, Nos. 21876132

\section{REFERENCES}

Ben, Y., Fu, C., Hu, M., Liu, L., Wong, M. H., and Zheng, C. (2019). Human health risk assessment of antibiotic resistance associated with antibiotic residues in the environment: a review. Environ. Res. 169, 483-493. doi: 10.1016/j.envres.2018.11.040

Berlina, A. N., Taranova, N. A., Zherdev, A. V., Vengerov, Y. Y., and Dzantiev, B. B. (2013). Quantum dot-based lateral flow immunoassay for detection of chloramphenicol in milk. Anal. Bioanal. Chem. 405, 4997-5000. doi: $10.1007 /$ s00216-013-6876-3

Chen, J., Xu, F., Jiang, H., Hou, Y., Rao, Q., Guo, P., et al. (2009). A novel quantum dot-based fluoroimmunoassay method for detection of Enrofloxacin residue in chicken muscle tissue. Food Chem. 113, 1197-1201. doi: 10.1016/j.foodchem.2008.08.006

Chen, T.-W., Rajaji, U., Chen, S.-M., Muthumariyappan, A., Mogren, M. M. A., Jothi Ramalingam, R., et al. (2019). Facile synthesis of copper(II) oxide nanospheres covered on functionalized multiwalled carbon nanotubes modified electrode as rapid electrochemical sensing platform for supersensitive detection of antibiotic. Ultrasonics Sonochem. 58, 104596-104596. doi: 10.1016/j.ultsonch.2019.05.013

Chen, X., Luan, Y., Wang, N., Zhou, Z., Ni, X., Cao, Y., et al. (2018). Ratiometric fluorescence nanosensors based on core-shell structured carbon/CdTe quantum dots and surface molecularly imprinted polymers for the detection of sulfadiazine. J. Separation Sci. 41, 4394-4401. doi: 10.1002/jssc.201800866

Coleman, J. N., Khan, U., Blau, W. J., and Gun'ko, Y. K. (2006). Small but strong: a review of the mechanical properties of carbon nanotube-polymer composites. Carbon 44,1624-1652. doi: 10.1016/j.carbon.2006.02.038

Dang, X., Zhao, H., Wang, X., Sailijiang, T., Chen, S., and Quan, X. (2018). Photoelectrochemical aptasensor for sulfadimethoxine using g-C3N4 quantum dots modified with reduced graphene oxide. Microchim. Acta 185:4. doi: 10.1007/s00604-018-2877-4

Ding, S., Chen, J., Jiang, H., He, J., Shi, W., Zhao, W., et al. (2006). Application of quantum dot-antibody conjugates for detection of sulfamethazine residue in chicken muscle tissue. J. Agri. Food Chem. 54, 6139-6142. doi: $10.1021 /$ jf0606961

Gleiter, H. (1989). Nanocrystalline materials. Progr. Mater. Sci. 33, 223-315. doi: 10.1016/0079-642590001-7

Gleiter, H. (2000). Nanostructured materials: basic concepts and microstructure. Acta Mater. 48, 1-29. doi: 10.1016/s1359-645400285-2

Gleiter, H., and Marquardt, P. (1984). Nanocrystalline structures - an approach to new materials. Zeitschrift Metallkunde 75, 263-267.

Govindasamy, M., Chen, S.-M., Mani, V., Devasenathipathy, R., Umamaheswari, R., Joseph Santhanaraj, K., et al. (2017). Molybdenum disulfide nanosheets coated multiwalled carbon nanotubes composite for highly sensitive determination of chloramphenicol in food samples milk, honey and powdered milk. J. Colloid Interface Sci. 485, 129-136. doi: 10.1016/j.jcis.2016. 09.029

Hassanzadeh, J., Moghadam, B. R., Sobhani-Nasab, A., Ahmadi, F., and RahimiNasrabadi, M. (2019). Specific fluorometric assay for direct determination of amikacin by molecularly imprinting polymer on high fluorescent g-C3N4 quantum dots. Spectrochim. Acta. 214, 451-458. doi: 10.1016/j.saa.2019.02.067

He, B., Wang, L., Dong, X., Yan, X., Li, M., Yan, S., et al. (2019). Aptamer-based thin film gold electrode modified with gold nanoparticles and carboxylated multiwalled carbon nanotubes for detecting oxytetracycline in chicken samples. Food Chem. 300, 125179-125179. doi: 10.1016/j.foodchem.2019.125179

He, B.-S., and Yan, S.-,s. (2018). Electrochemical aptasensor based on aptamercomplimentary strand conjugate and thionine for sensitive detection of and 21577100), the National Program for Support of Top-Notch Young Professionals, Shanghai Rising-Star Program (18QA1404300), the Fundamental Research Funds for the Central Universities (22120180102), and Young Excellent Talents in Tongji University (2015KJ001), Science and Technology Commission of Shanghai Municipality (18DZ1204400). tetracycline with multi-walled carbon nanotubes and gold nanoparticles amplification. Anal. Methods 10, 783-790. doi: 10.1039/c7ay02728a

Hou, M., Xiong, L., and Liu, G. (2011). Determination of sparfloxacin with fluorescence probe of CdSe/CdS quantum dots. Phys. Test. Chem. Anal. 47, 1387-1390. doi: 10.1016/j.jlumin.2014.08.006

Huang, S., Gan, N., Li, T., Zhou, Y., Cao, Y., and Dong, Y. (2018). Electrochemical aptasensor for multi-antibiotics detection based on endonuclease and exonuclease assisted dual recycling amplification strategy. Talanta 179, 28-36. doi: 10.1016/j.talanta.2017.10.016

Khataee, A. R., Hasanzadeh, A., Iranifam, M., Fathinia, M., Hanifehpour, Y., and Joo, S. W. (2014). CuO nanosheets-enhanced flow-injection chemiluminescence system for determination of vancomycin in water, pharmaceutical and human serum. Spectrochim. Acta 122, 737-743. doi: 10.1016/j.saa.2013.12.014

Kokulnathan, T., and Chen, S.-M. (2020). Robust and selective electrochemical detection of antibiotic residues: the case of integrated lutetium vanadate/graphene sheets architectures. J. Hazardous Mater. 384, 121304-121304. doi: 10.1016/j.jhazmat.2019.121304

Kuemmerer, K. (2009). Antibiotics in the aquatic environment-a review-part I. Chemosphere 75,417-434. doi: 10.1016/j.chemosphere.2008.11.086

Leptihn, S., Guo, L., Frecer, V., Ho, B., Ding, J. L., and Wohland, T. (2010). Single molecule resolution of the antimicrobial action of quantum dot-labeled sushi peptide on live bacteria. Virulence 1, 42-44. doi: 10.4161/viru.1.1.10229

Li, Z., Liu, C., Sarpong, V., and Gu, Z. (2019). Multisegment nanowire/nanoparticle hybrid arrays as electrochemical biosensors for simultaneous detection of antibiotics. Biosensors Bioelectr. 126, 632-639. doi: 10.1016/j.bios.2018.10.025

Liu, X., Hu, M., Wang, M., Song, Y., Zhou, N., He, L., et al. (2019). Novel nanoarchitecture of Co-MOF-on-TPN-COF hybrid: ultralowly sensitive bioplatform of electrochemical aptasensor toward ampicillin. Biosens. Bioelectr. 123, 59-68. doi: 10.1016/j.bios.2018.09.089

Liu, X., Zhou, Z., Wang, T., Xu, Y., Lu, K., and Yan, Y. (2020). Molecularly imprinted polymers-captivity $\mathrm{ZnO}$ nanorods for sensitive and selective detecting environmental pollutant. Spectrochim. Acta A Mol Biomol Spectr. 228:e117785. doi: 10.1016/j.saa.2019.117785

Liu, Z., Liu, S., Wang, L., Peng, J., and He, Y. (2009). Resonance Rayleigh scattering and resonance non-linear scattering method for the determination of aminoglycoside antibiotics with water solubility CdS quantum dots as probe. Spectrochim. Acta A Mol. Biomol. Spectr. 74, 36-41. doi: 10.1016/j.saa.2009.04.026

Malik, R., Pinnaka, A. K., Kaur, M., Kumar, V., Tikoo, K., Singh, S., et al. (2019). Water-soluble glutathione-CdS QDs with exceptional antimicrobial properties synthesizedviagreen route for fluorescence sensing of fluoroquinolones. J. Chem. Technol. Biotechnol. 94, 1082-1090. doi: 10.1002/jctb. 5855

Mohammad-Razdari, A., Ghasemi-Varnamkhasti, M., Izadi, Z., Ensafi, A. A., Rostami, S., and Siadat, M. (2019a). An impedimetric aptasensor for ultrasensitive detection of Penicillin $G$ based on the use of reduced graphene oxide and gold nanoparticles. Mikrochim Acta 186, 372-372. doi: 10.1007/s00604-019-3510-x

Mohammad-Razdari, A., Ghasemi-Varnamkhasti, M., Izadi, Z., Rostami, S., Ensafi, A. A., Siadat, M., et al. (2019b). Detection of sulfadimethoxine in meat samples using a novel electrochemical biosensor as a rapid analysis method. J. Food Composition Anal. 82:e103252. doi: 10.1016/j.jfca.2019.103252

Munawar, A., Tahir, M. A., Shaheen, A., Lieberzeit, P. A., Khan, W. S., and Bajwa, S. Z. (2018). Investigating nanohybrid material based on 3D CNTs@Cu nanoparticle composite and imprinted polymer for highly 
selective detection of chloramphenicol. J. Hazardous Mater. 342, 96-106. doi: 10.1016/j.jhazmat.2017.08.014

Netea, S. A., Messina, N. L., and Curtis, N. (2019). Early-life antibiotic exposure and childhood food allergy: a systematic review. J. Allergy Clin. Immunol. 144:1445. doi: 10.1016/j.jaci.2019.08.001

Peng, J., Huang, Q., Zhuge, W., Liu, Y., Zhang, C., Yang, W., et al. (2018). Blue-light photoelectrochemical sensor based on nickel tetra-amined phthalocyaninegraphene oxide covalent compound for ultrasensitive detection of erythromycin. Biosens. Bioelectr. 106, 212-218. doi: 10.1016/j.bios.2018.02.009

Qie Gen, L., Yuan Fang, L., and Cheng Zhi, H. (2007). A light scattering and fluorescence emission coupled ratiometry using the interaction of functional CdS quantum dots with aminoglycoside antibiotics as a model system. Talanta 71, 567-572. doi: 10.1016/j.talanta.2006.04.035

Qin, Y., Ma, Y., Jin, X., Zhang, L., Ye, G., and Zhao, S. (2015). A sensitive fluorescence turn-on assay of bleomycin and nuclease using WS2 nanosheet as an effective sensing platform. Anal. Chim. Acta 866, 84-89. doi: 10.1016/j.aca.2015.01.049

Rastgar, S., and Shahrokhian, S. (2014). Nickel hydroxide nanoparticles-reduced graphene oxide nanosheets film: layer-by-layer electrochemical preparation, characterization and rifampicin sensory application. Talanta 119, 156-163. doi: 10.1016/j.talanta.2013.10.047

Rosati, G., Ravarotto, M., Scaramuzza, M., De Toni, A., and Paccagnella, A. (2019). Silver nanoparticles inkjet-printed flexible biosensor for rapid label-free antibiotic detection in milk. Sens. Actuators. Sensors and Actuators B-Chem. 280, 280-289. doi: 10.1016/j.snb.2018.09.084

Roushani, M., and Ghanbari, K. (2018). A novel aptasensor based on gold nanorods/ZnS QDs-modified electrode for evaluation of streptomycin antibiotic. Anal. Methods 10, 5197-5204. doi: 10.1039/c8ay01815d

Roushani, M., Rahmati, Z., Farokhi, S., Hoseini, S. J., and Fath, R. H. (2020). The development of an electrochemical nanoaptasensor to sensing chloramphenicol using a nanocomposite consisting of graphene oxide functionalized with (3-Aminopropyl) triethoxysilane and silver nanoparticles. Mater. Sci. Eng. C Mater. Biol. Appl. 108, 110388-110388. doi: 10.1016/j.msec.2019.110388

Shad, N. A., Bajwa, S. Z., Amin, N., Taj, A., Hameed, S., Khan, Y., et al. (2019). Solution growth of 1D zinc tungstate (ZnWO4) nanowires; design, morphology, and electrochemical sensor fabrication for selective detection of chloramphenicol. J. Hazard. Mater. 367, 205-214. doi: 10.1016/j.jhazmat.2018.12.072

Tian, Q., Wang, Y., Deng, R., Lin, L., Liu, Y., and Li, J. (2015). Carbon nanotube enhanced label-free detection of microRNAs based on hairpin probe triggered solid-phase rolling-circle amplification. Nanoscale 7, 987-993. doi: $10.1039 / \mathrm{c} 4 \mathrm{nr} 05243 \mathrm{a}$

Vega, D., Agui, L., Gonzalez-Cortes, A., Yanez-Sedeno, P., and Pingarron, J. M. (2007). Voltammetry and amperometric detection of tetracyclines at multiwall carbon nanotube modified electrodes. Anal. Bioanal. Chem. 389, 951-958. doi: 10.1007/s00216-007-1505-7

Velusamy, V., Palanisamy, S., Kokulnathan, T., Chen, S. W., Yang, T. C. K., Banks, C. E., et al. (2018). Novel electrochemical synthesis of copper oxide nanoparticles decorated graphene-beta-cyclodextrin composite for trace-level detection of antibiotic drug metronidazole. J. Colloid Interface Sci. 530, 37-45. doi: 10.1016/j.jcis.2018.06.056

Wang, C., Li, Q., Wang, B., Li, D., and Yu, J. (2018). Fluorescent sensors based on AIEgen-functionalised mesoporous silica nanoparticles for the detection of explosives and antibiotics. Inorg. Chem. Front. 5, 2183-2188. doi: 10.1039/c8qi00622a

Wang, L., Peng, J., Liu, Z., and He, Y. (2010). Resonance Rayleighscattering spectral method for the determination of some aminoglycoside antibiotics using CdTe quantum dots as a probe. Luminescence 25, 424-430. doi: 10.1002/bio.1170

Wang, Y., Bian, F., Qin, X., and Wang, Q. (2018). Visible light photoelectrochemical aptasensor for chloramphenicol by using a $\mathrm{TiO}_{2}$ nanorod array sensitized with $\mathrm{Eu}(\mathrm{III})$-doped CdS quantum dots. Mikrochim. Acta 185, 161-161. doi: 10.1007/s00604-018-2711-z
Wang, Y., Lu, J., Tang, L., Chang, H., and Li, J. (2009). Graphene oxide amplified electrogenerated chemiluminescence of quantum dots and its selective sensing for glutathione from thiol-containing compounds. Anal. Chem. 81, 9710-9715. doi: $10.1021 /$ ac901935a

Wang, Y., Ma, T., Ma, S., Liu, Y., Tian, Y., Wang, R., et al. (2017). Fluorometric determination of the antibiotic kanamycin by aptamerinduced FRET quenching and recovery between $\mathrm{MoS}_{2}$ nanosheets and carbon dots. Microchim. Acta 184, 203-210. doi: 10.1007/s00604-0162011-4

Wei, Q., Mao, K., Wu, D., Dai, Y., Yang, J., Du, B., et al. (2010). A novel label-free electrochemical immunosensor based on graphene and thionine nanocomposite. Sensors Actuators B Chem. 149, 314-318. doi: $10.1016 / j . s n b .2010 .06 .008$

Wu, C., Cheng, R., Wang, J., Wang, Y., Jing, X., Chen, R., et al. (2018), Fluorescent molecularly imprinted nanoparticles for selective and rapid detection of ciprofloxacin in aquaculture water. J. Separation Sci. 41, 3782-3790. doi: $10.1002 /$ jssc. 201800418

Xiao, F., Zhao, F., Li, J., Yan, R., Yu, J., and Zeng, B. (2007). Sensitive voltammetric determination of chloramphenicol by using single-wall carbon nanotube-gold nanoparticle-ionic liquid composite film modified glassy carbon electrodes. Anal. Chim. Acta 596, 79-85. doi: 10.1016/j.aca.2007.05.053

Xu, J., Yu, C., Feng, T., Liu, M., Li, F., Wang, Y., et al. (2018). N-Carbamoylmaleimide-treated carbon dots: stabilizing the electrochemical intermediate and extending it for the ultrasensitive detection of organophosphate pesticides. Nanoscale 10, 19390-19398. doi: 10.1039/c8nr05098h

Yu, C., Yu, J., Zhang, H., He, Z., Sha, Y., Liu, B., et al. (2019). A facile approach for rapid on-site screening of nicotine in natural tobacco. Environ. Pollution 259:113841. doi: 10.1016/j.envpol.2019.113841

Zang, S., Liu, Y., Lin, M., Kang, J., Sun, Y., and Lei, H. (2013). A dual amplified electrochemical immunosensor for ofloxacin: polypyrrole film-Au nanocluster as the matrix and multi-enzyme-antibody functionalized gold nanorod as the label. Electrochim. Acta 90, 246-253. doi: 10.1016/j.electacta.2012. 12.021

Zeng, R., Tang, Y., Zhang, L., Luo, Z., and Tang, D. (2018). Dual-readout aptasensing of antibiotic residues based on gold nanocluster-functionalized $\mathrm{MnO} 2$ nanosheets with target-induced etching reaction. J. Mater. Chem. B 6 , 8071-8077. doi: 10.1039/c8tb02642d

Zengin, A., Tamer, U., and Caykara, T. (2014). Extremely sensitive sandwich assay of kanamycin using surface-enhanced Raman scattering of 2mercaptobenzothiazole labeled gold@silver nanoparticles. Anal. Chim. Acta 817, 33-41. doi: 10.1016/j.aca.2014.01.042

Zhang, K., Lu, L., Wen, Y., Xu, J., Duan, X., Zhang, L., et al. (2013). Facile synthesis of the necklace-like graphene oxide-multi-walled carbon nanotube nanohybrid and its application in electrochemical sensing of azithromycin. Anal. Chim. Acta 787, 50-56. doi: 10.1016/j.aca.2013.05.037

Zhang, Y., and Wei, Q. (2016). The role of nanomaterials in electroanalytical biosensors: a mini review. J. Electroanal. Chem. 781, 401-409. doi: 10.1016/j.jelechem.2016.09.011

Zhu, K., Li, J., Wang, Z., Jiang, H., Beier, R. C., Xu, F., et al. (2011) Simultaneous detection of multiple chemical residues in milk using broadspecificity antibodies in a hybrid immunosorbent assay. Biosens. Bioelectr. 26, 2716-2719. doi: 10.1016/j.bios.2010.09.01

Conflict of Interest: The authors declare that the research was conducted in the absence of any commercial or financial relationships that could be construed as a potential conflict of interest.

Copyright $\odot 2020$ Dong, Li and Wang. This is an open-access article distributed under the terms of the Creative Commons Attribution License (CC BY). The use, distribution or reproduction in other forums is permitted, provided the original author(s) and the copyright owner(s) are credited and that the original publication in this journal is cited, in accordance with accepted academic practice. No use, distribution or reproduction is permitted which does not comply with these terms. 